\title{
The impact of corticosteroid use on inpatients with inflammatory bowel disease and positive polymerase chain reaction for Clostridium difficile
}

\author{
Huei-Wen Lim ${ }^{1}$, Isaiah P. Schuster ${ }^{2}$, Ramona Rajapakse ${ }^{2}$, Farah Monzur², Sundas Khan ${ }^{3}$, Keith Sultan ${ }^{1}$ \\ ${ }^{I}$ Division of Gastroenterology and Hepatology, Donald and Barbara Zucker School of Medicine at Hofstra/Northwell, Manhasset, NY; ${ }^{2}$ Division \\ of Gastroenterology and Hepatology, Stony Brook University Hospital, Stony Brook, NY; ${ }^{3}$ Division of Gastroenterology and Hepatology, The \\ Feinstein Institute for Medical Research, Manhasset, NY, USA
}

Background/Aims: Optimal management of inflammatory bowel disease (IBD) with concomitant Clostridium difficile infection (CDI) is controversial, especially when CDI diagnosis is made by polymerase chain reaction (PCR) testing, which may reflect colonization without infection. Methods: We performed a multicenter review of all inpatients with IBD and PCR diagnosed CDI. Outcomes included length of stay, 30- and 90-day readmission, colectomy during admission and within 3 months, intensive care unit (ICU) admission, CDI relapse and death for patients who received corticosteroid (CS) after CDI diagnosis versus those that did not. Propensity-adjusted regression analysis of outcomes based on CS usage was performed. Results: We identified 177 IBD patients with CDI, 112 ulcerative colitis and 65 Crohn's disease. For IBD overall, CS after CDI diagnosis was associated with prolonged hospitalization ( 5.5 days: $95 \%$ confidence interval [CI], $1.5-9.6$ days; $P=0.008$ ), higher colectomy rate within 3 months (odds ratio [OR], 5.5; 95\% CI, 1.1-28.2; $P=0.042)$ and more frequent ICU admissions (OR, 7.8; 95\% CI, 1.5-41.6; $P=0.017)$ versus no CS. CS use post-CDI diagnosis in UC patients was associated with prolonged hospitalization (6.2 days: $95 \% \mathrm{CI}, 0.4-12.0$ days; $P=0.036)$ and more frequent ICU admissions (OR, 7.4; 95\% CI, 1.1-48.7; $P=0.036)$. Conclusions: CS use among IBD inpatients with CDI diagnosed by PCR is associated with poorer outcomes and would seem to reinforce the importance of $C$. difficile toxin assay to help distinguish colonization from infection. This adverse effect appears more prominent among those with UC. (Intest Res 2019;17:244-252)

Key Words: Inflammatory bowel disease; Corticosteroids; Clostridium difficile; Adverse outcomes; Polymerase chain reaction

\section{INTRODUCTION}

Inflammatory bowel disease (IBD), mainly including CD and UC, is a condition caused by a dysregulated immune response to non-pathogenic gut microflora resulting in spontaneous and chronic inflammation of the bowel. ${ }^{1-3}$ Clostridium difficile

Received July 2, 2018. Revised November 10, 2018.

Accepted November 12, 2018.

Correspondence to Huei-Wen Lim, Division of Gastroenterology and

Hepatology, Donald and Barbara Zucker School of Medicine at Hofstra/

Northwell, 300 Community Drive, Manhasset, NY 11030, USA. Tel: +1-267-

353-4044, Fax: +1-516-562-3555, E-mail: lim.hueiwen@gmail.com

ORCID Huei-Wen Lim (https://orcid.org/0000-0002-6745-2277) is a spore-forming gram-positive anaerobic bacterium which may be responsible for a spectrum of clinical presentations ranging from asymptomatic colonization, to diarrhea with colitis, fulminant colitis, recurrent disease and death. ${ }^{4-6}$ C. difficile infection (CDI) is increasing in prevalence and severity, with increased rates of posttreatment relapse described ${ }^{7,8}$ In 2011, approximately 453,000 cases of CDI were reported in the United States and it is currently the most common nosocomial infection found in U.S. hospitals. ${ }^{9}$ CDI has also been increasingly associated with IBD flares. ${ }^{10}$ Based on data from the Nationwide Inpatient Sample, there was a doubling (1.4\% to $2.9 \%$ ) in IBD hospitalizations that were complicated by CDI from 1998 
to 2007 and CDI has consistently been associated with poor clinical outcomes, such as an increase in the number and length of hospital stay, higher rates of colectomy, and a 4-fold increase in mortality. ${ }^{11-15}$ As such, it is recommended by current guidelines that all patients with IBD hospitalized with a disease flare undergo testing for CDI and be treated with antibiotics when testing is positive. ${ }^{10}$

Given the similar presentations, IBD flare may be clinically indistinguishable from CDI. Treatment of IBD with CDI presents a clinical dilemma, as IBD therapies may need to continue or even be intensified to treat the underlying disease, but may result in worse outcomes in those with concurrent CDI. ${ }^{16}$ Many IBD therapies such as corticosteroids (CS), immunomodulators such as the thiopurines and methotrexate, and biologic therapies have themselves been implicated as possible risk factors for CDI. ${ }^{17,18}$ There is currently no consensus regarding the safety of continued or escalated immune therapy in such a setting.

Most prior investigations of IBD and CDI outcomes have diagnosed CDI by enzyme immunoassay (EIA) testing for toxin $\mathrm{A} / \mathrm{B}{ }^{16}$ The expanding use of more sensitive testing for $C$. difficile by PCR complicates the clinical dilemma further, as a positive PCR result alone may reflect colonization without true infection. ${ }^{19,20}$ While current guidelines advocate a 2 -step process using toxin assay following a positive PCR to distinguish infection from colonization, not all institutions follow such a protocol. $^{21,22}$ Even endoscopic evaluation is unreliable as a guide to therapy for $C$. difficile complicating IBD, as the mucosa generally fails to show the typical pseudomembranes, and biopsies inconsistently show the fibrin eruptions classically associated with CDI on histology. ${ }^{17}$ In non-IBD populations C. difficile diagnosed by PCR alone has not consistently been associated with negative outcomes, but it is unknown if this is also true for the IBD population. ${ }^{23}$

For the management of inpatients with IBD exacerbation, CS remain a mainstay of acute therapy. To date, no studies have investigated whether CDI diagnosed by high sensitivity PCR with IBD carries the same risk for adverse outcomes as CDI diagnosed by EIA testing during hospitalization for IBD exacerbation. Our goal was to analyze the effect of CS use on the clinical outcomes in IBD inpatients with CDI diagnosed by PCR alone.

\section{METHODS}

The study was approved by the Northwell Health System and
Stony Brook Institutional Review Boards (IRB No. HS16-0306). The study population was defined as all patients 18 years of age or older hospitalized with a diagnosis of IBD and concomitant PCR-diagnosed CDI at 7 institutions across the Northwell Health system ( 2 tertiary care hospitals and 5 community hospitals) and Stony Brook University Hospital from 2011 to 2016 identified via Sunrise and Cerner PowerChart, the centralized clinical data registry for Northwell Health and Stony Brook University Hospital respectively. While a positive PCR alone may indicate colonization without infection, the study marks the period of time in which 1-step PCR testing without additional EIA toxin assay was the standard method of CDI diagnosis at all of the institutions studied, and the results reflect this method of diagnosis. During the study period laboratory protocol specified that only stools without any formation, Bristol Stool Scale 6 or 7, would undergo PCR testing which is a common method of excluding potential cases of $C$. difficile colonization without infection from PCR analysis. Patients' medical records from inpatient admissions, emergency room visits, operative notes and pathology reports were linked to obtain longitudinal data. Patients who had IBD and PCR-diagnosed CDI were identified by a systematic search of discharge diagnosis of admitted patients as well as laboratory database of positive PCR diagnosed CDI and confirmed via medical record chart review.

\section{Study Definitions}

Patients admitted with IBD and PCR-diagnosed CDI were stratified into 2 groups: patients who received CS post-CDI diagnosis and patients who did not receive CS post-CDI diagnosis. CS use post-CDI diagnosis was defined as any dose of CS use in the inpatient setting after the diagnosis of CDI and given for at least 3 days. Pre-CDI diagnosis CS use and inpatient immunomodulator/biologic use were also evaluated for their effect on adverse outcomes. Pre-CDI diagnosis CS use was defined as any dose used in the outpatient or inpatient setting prior to the diagnosis of CDI. Pre-admission immunomodulator/biologic therapy was defined by patient reported active use of 6-mercaptopurine, azathioprine, methotrexate, infliximab, adalimumab, certolizumab, golimumab, vedolizumab or ustekinumab in their admission medications list. Inpatient immunomodulator/biologic therapy was defined by the use of 6-mercaptopurine, azathioprine, methotrexate, infliximab, adalimumab, certolizumab, golimumab, vedolizumab or ustekinumab documented as ordered in the hospital record. Demographic data such as age, gender and disease type, $\mathrm{CD}$ ver- 
sus UC were collected. Given the absence of validated clinical or endoscopic disease severity indices for CD or UC from the medical record, surrogate demographic and clinical markers previously used in retrospective studies to assess colitis severity such as patient's age, BMI, albumin, creatinine, white blood cell count and hypotension (defined as systolic blood pressure $<100 \mathrm{mmHg}$ ) within 48 hours of admission were collected.

\section{Study Outcomes}

The outcomes assessed were: length of stay, 30- and 90-day readmission with IBD flare or CDI symptoms defined as having diarrhea, abdominal pain or blood in stool, colectomy within 3 months of admission, intensive care unit (ICU) admission during initial admission, colon perforation requiring colectomy during the same hospitalization, CDI relapse defined as re-hospitalization within 3 months of previous admission with CDI, and death. Patients who underwent colectomies were censored for readmission outcomes as patients could not be readmitted for symptoms of colitis post-colectomy. Outcomes were evaluated for the entire cohort and then stratified by disease UC and CD respectively.

\section{Statistical Analysis}

Statistical analyses were performed using Intercooled Stata software, version 12.0 (StataCorp, College Station, TX, USA). Fisher exact test was used for comparisons of categorical variables. $t$-test and Wilcoxon rank-sum were used for comparisons of continuous outcomes for parametric and non-parametric distributed data respectively. Propensity scores for each patient to receive post-CDI diagnosis CS were generated via a model adjusting for patients' age, BMI, admission albumin, creatinine, white blood count levels and presence of hypotension. These variables were selected based on statistical significance on univariate analysis and on clinical face validity. The propensity score was then used in regression models for adjusted analyses. Logistic and linear regressions were used for adjusted analyses of categorical and continuous endpoints respectively. Different propensity scores were generated and used for the subset analyses of outcomes based on pre-CDI diagnosis CS use and inpatient immunomodulator/biologic use. Statistical significance was accepted when the 2-sided test had a $P$-value of $<0.05$.

Table 1. Demographic and Clinical Characteristics of the Study Population

\begin{tabular}{|c|c|c|c|c|}
\hline Characteristic & $\begin{array}{l}\text { All patients } \\
(n=177)\end{array}$ & $\begin{array}{c}\text { Received CS } \\
\text { post-CDI diagnosis } \\
(n=64)\end{array}$ & $\begin{array}{l}\text { Did not receive CS } \\
\text { post-CDI diagnosis } \\
(n=113)\end{array}$ & $P$-value \\
\hline Age (yr) & $46(28-67)$ & $38(23-62)$ & $53(32-71)$ & $0.003^{a}$ \\
\hline Female sex & 108 (61.02) & 38 (59.38) & 70 (61.95) & 0.736 \\
\hline $\operatorname{BMI}\left(\mathrm{kg} / \mathrm{m}^{2}\right)$ & $24(20-28)$ & $24(20-28)$ & $24(20-28)$ & 0.797 \\
\hline UC & $112(63.28)$ & $44(68.75)$ & $68(60.18)$ & 0.330 \\
\hline$C D$ & $65(36.72)$ & $20(31.25)$ & $45(39.82)$ & 0.330 \\
\hline Race & & & & 0.969 \\
\hline White & $95(53.67)$ & $35(54.69)$ & $60(53.10)$ & \\
\hline African American & $12(6.78)$ & $4(6.25)$ & 8 (7.08) & \\
\hline Asian & $11(6.21)$ & $4(6.25)$ & $7(6.19)$ & \\
\hline Other/multiracial & $59(33.33)$ & $21(32.81)$ & $38(33.63)$ & \\
\hline Proton pump inhibitor $/ \mathrm{H}_{2}$ blocker on prior month & $43(24.29)$ & $14(21.88)$ & $29(25.66)$ & 0.572 \\
\hline Immunomodulator/biologic used in prior month & $68(38.42)$ & $24(37.50)$ & $44(38.94)$ & 0.850 \\
\hline Inpatient immunomodulator/biologic use & $72(40.68)$ & $33(51.56)$ & $39(34.51)$ & $0.027^{\mathrm{a}}$ \\
\hline Pre-admit CS use & $32(18.08)$ & $20(31.25)$ & $12(10.62)$ & $0.001^{\mathrm{a}}$ \\
\hline Pre-CDI diagnosis CS use & $76(42.94)$ & $53(82.81)$ & $23(20.35)$ & $0.000^{\mathrm{a}}$ \\
\hline Time to CDI diagnosis (day) & $1(1-2)$ & $2(1-3)$ & $1(1-2)$ & 0.987 \\
\hline
\end{tabular}

Values are presented as median (interquartile range) or number (\%).

${ }^{a}$ Significant $P$-value.

CS, corticosteroid; CDI, Clostridium difficile infection. 


\section{RESULTS}

\section{Demographics/Characteristics of Study Population}

We identified 177 patients with IBD and PCR diagnosed CDI (154 from the 7 Northwell Health institutions and 23 from Stony Brook University Hospital) from 2011 to 2016, 61\% female and approximately two-thirds with UC (Table 1). Only 32 patients (18.08\%) had documented CS use prior to hospitalization, 20 of whom continued CS post-CDI diagnosis. Seventy-six patients (42.94\%) received CS before CDI diagnosis, and 64 (36.16\%) received CS following CDI diagnosis. Patients received systemic CS either as prednisone, methylprednisolone, dexamethasone or hydrocortisone. Of the 64 patients receiving CS following CDI diagnosis, dose ranges converted into hydrocortisone equivalent doses (HED) ranged from 20 $\mathrm{mg}$ to $750 \mathrm{mg}$. The most common regimen, used in 17 patients, was prednisone $40 \mathrm{mg}$ daily, or $160 \mathrm{mg}$ HED, and the average CS dose was $216 \mathrm{mg}$ HED. Of the patients who received CS following a CDI diagnosis, 53 were patients continuing CS ordered prior to CDI diagnosis. Only 11 patients were newly pre- scribed CS following a CDI diagnosis, 5 of whom began CS either on the same day or the day following positive PCR testing. Sixty-eight patients (38.42\%) had pre-admission immunomodulator or biologic therapy usage. Patients who received CS after the diagnosis of CDI were significantly younger $(P=0.003)$ and were more likely to receive immunomodulatory/biologic therapy while hospitalized $(P=0.027)$ compared to those not receiving CS after CDI diagnosis, but were similar by gender and ethnicity (Table 1).

During hospitalization 37 patients (21\%) had endoscopic evaluation, of which 18 had colonoscopy and 19 had flexible sigmoidoscopy. Among the clinical parameters of disease severity analyzed, patients not receiving CS following CDI diagnosis were more likely to have a creatinine level greater than $1.3 \mathrm{mg} / \mathrm{dL}(P=0.026)$, but other clinical parameters were similar to those receiving CS following CDI diagnosis (Table 2). The median time to CDI diagnosis following admission was one day, and there was no significant difference in time following admission to $C$. difficile diagnosis for those who did and did not receive CS $(P=0.987)$. All patients received treatment for

Table 2. Clinical Parameters Indicating Severity at Presentation of the Study Population

\begin{tabular}{|c|c|c|c|c|}
\hline Parameter & $\begin{array}{l}\text { All patients } \\
(n=177)\end{array}$ & $\begin{array}{l}\text { Received CS post-CDI } \\
\text { diagnosis }(n=64)\end{array}$ & $\begin{array}{c}\text { Did not receive CS post-CDI } \\
\text { diagnosis }(n=113)\end{array}$ & $P$-value \\
\hline Leukocytosis $>15 \times 10^{3}$ cells $/ \mu \mathrm{L}$ & $61(34.46)$ & $34(53.13)$ & $27(23.89)$ & 0.563 \\
\hline Hypoalbuminemia $<2.5 \mathrm{~g} / \mathrm{dL}$ & $31(17.51)$ & $10(15.63)$ & $21(18.58)$ & 0.614 \\
\hline Elevated creatinine $>1.3 \mathrm{mg} / \mathrm{dL}$ & $35(19.77)$ & $7(10.94)$ & $28(24.78)$ & $0.026^{a}$ \\
\hline Systolic blood pressure $<100 \mathrm{mmHg}$ & $73(41.24)$ & $24(37.50)$ & $49(43.36)$ & 0.651 \\
\hline
\end{tabular}

Values are presented as number (\%).

asignificant $P$-value.

CS, corticosteroid; CDI, Clostridium difficile infection.

Table 3. Unadjusted Outcomes Analysis for All Patients

\begin{tabular}{|c|c|c|c|c|}
\hline Outcome & $\begin{array}{l}\text { All patients } \\
(n=177)\end{array}$ & $\begin{array}{l}\text { Received CS post-CDI } \\
\text { diagnosis }(n=64)\end{array}$ & $\begin{array}{l}\text { Did not receive CS post-CDI } \\
\text { diagnosis }(n=113)\end{array}$ & $P$-value \\
\hline Length of stay in days & $7(4-11)$ & $8(5-16)$ & $6(3-9)$ & $0.001^{\mathrm{a}}$ \\
\hline 30-Day readmission & $16(9.04)$ & $4(6.25)$ & $12(10.62)$ & 0.407 \\
\hline 90-Day readmission & $28(15.82)$ & $7(10.94)$ & $21(18.58)$ & 0.252 \\
\hline Colectomy within 3 months of admission & $14(7.91)$ & $8(12.50)$ & $6(5.31)$ & 0.089 \\
\hline ICU admission & $14(7.91)$ & $9(14.06)$ & $5(4.42)$ & $0.007^{\mathrm{a}}$ \\
\hline Colon perforation & $3(1.69)$ & $3(4.69)$ & 0 & $0.020^{\mathrm{a}}$ \\
\hline CDI relapse & $26(14.69)$ & $7(10.94)$ & $19(16.81)$ & 0.289 \\
\hline Death & $5(2.82)$ & $2(3.13)$ & $3(2.65)$ & 0.856 \\
\hline
\end{tabular}

Values are presented as median (interquartile range) or number (\%).

${ }^{a}$ Significant $P$-value.

CS, corticosteroid; CDI, Clostridium difficile infection; ICU, intensive care unit 
CDI following their positive PCR test, and there was no difference in antibiotic utilization for oral metronidazole $(P=0.997)$, fidaxomicin $(P=0.292)$ or vancomycin $(P=0.969)$ between those receiving/continuing and not receiving CS following CDI diagnosis.

\section{Adverse Outcomes}

\section{1) Unadjusted Outcome Analysis for All Patients}

The median length of stay for all patients was 7 days (interquartile range, 4-11 days). On the unadjusted analysis, median length of stay for patients who received CS post-CDI diagnosis was 8 days compared to 6 days in patients who did not receive CS post-CDI diagnosis $(P=0.001)$. There was also a significant increase in number of ICU admissions $(P=0.007)$ and colon perforations $(P=0.020)$ in the group that received
CS post-CDI diagnosis. All patients with colon perforations underwent colectomy. Other adverse outcomes were similar between the 2 groups (Table 3 ).

\section{2) Unadjusted Outcome Analysis for UC Patients}

Median length of stay for UC patients who received CS postCDI diagnosis was 8 days compared to 5 days in patients who did not receive CS post-CDI diagnosis $(P=0.048)$. There was also a greater number of colectomies within 3 months of admission $(P=0.009)$, ICU admissions $(P=0.007)$ and colon perforations $(P=0.028)$ in patients who received CS post-CDI diagnosis $(P=0.009)$ compared to patients who did not receive CS post-CDI diagnosis. Other adverse outcomes were similar between the 2 groups (Table 4 ).

Table 4. Unadjusted Outcome Analysis for UC Patients

\begin{tabular}{|c|c|c|c|c|}
\hline Outcome & $\begin{array}{l}\text { All UC patients } \\
\quad(n=112)\end{array}$ & $\begin{array}{l}\text { Received CS post-CDI } \\
\text { diagnosis }(n=44)\end{array}$ & $\begin{array}{c}\text { Did not receive CS post-CDI } \\
\text { diagnosis }(n=68)\end{array}$ & $P$-value \\
\hline Length of stay in days & $8(4-12)$ & $8(5-18)$ & $7(4-10)$ & $0.048^{\mathrm{a}}$ \\
\hline 30-Day readmission & $8(7.14)$ & $3(6.82)$ & $5(7.35)$ & 0.919 \\
\hline 90-Day readmission & 15 (13.39) & $5(11.36)$ & $10(14.71)$ & 0.252 \\
\hline Colectomy within 3 months of admission & $7(6.25)$ & $6(13.64)$ & $1(1.47)$ & $0.009^{a}$ \\
\hline ICU admission & $11(9.82)$ & $7(15.91)$ & $4(5.88)$ & $0.007^{\mathrm{a}}$ \\
\hline Colon perforation & $3(2.68)$ & $3(6.82)$ & 0 & $0.028^{a}$ \\
\hline CDI relapse & $18(16.07)$ & $5(11.36)$ & $13(19.12)$ & 0.290 \\
\hline Death & $5(4.46)$ & $2(4.55)$ & $3(4.41)$ & 0.960 \\
\hline
\end{tabular}

Values are presented as median (interquartile range) or number (\%).

${ }^{a}$ Significant $P$-value.

CS, corticosteroid; CDI, Clostridium difficile infection; ICU, intensive care unit.

Table 5. Unadjusted Outcome Analysis for CD patients

\begin{tabular}{|c|c|c|c|c|}
\hline Outcome & $\begin{array}{l}\text { All CD patients } \\
(n=65)\end{array}$ & $\begin{array}{l}\text { Received CS post-CDI } \\
\text { diagnosis }(n=20)\end{array}$ & $\begin{array}{c}\text { Did not receive CS post-CDI } \\
\text { diagnosis }(n=45)\end{array}$ & $P$-value \\
\hline Length of stay in days & $10(6-17)$ & $7.5(5-14)$ & $5(3-8)$ & $0.015^{\mathrm{a}}$ \\
\hline 30-day readmission & $8(12.31)$ & $1(5.00)$ & $7(15.56)$ & 0.234 \\
\hline 90-day readmission & $13(20.00)$ & $2(10.00)$ & $11(24.44)$ & 0.180 \\
\hline Colectomy within 3 months of admission & $7(10.77)$ & $2(10.00)$ & $5(11.11)$ & 0.916 \\
\hline ICU admission & $3(4.62)$ & $2(10.00)$ & $1(2.22)$ & 0.161 \\
\hline Colon perforation & 0 & 0 & 0 & NA \\
\hline CDI relapse & 8 (12.31) & $2(10.00)$ & $6(13.33)$ & 0.728 \\
\hline Death & 0 & 0 & 0 & NA \\
\hline
\end{tabular}

Values are presented as median (interquartile range) or number (\%).

${ }^{a}$ Significant $P$-value.

CS, corticosteroid; CDI, Clostridium difficile infection; ICU, intensive care unit; NA, not available. 


\section{3) Unadjusted Outcome Analysis for CD Patients}

Median length of stay for CD patients who received CS postCDI diagnosis was 7.5 days compared to 5 days in patients who did not receive CS post-CDI diagnosis $(P=0.015)$. Other adverse outcomes were similar between the 2 groups (Table 5).

\section{4) Propensity-Adjusted Regression Analysis}

CS use post-CDI diagnosis was associated with prolonged hospitalization (5.5 days: $95 \% \mathrm{CI}, 1.5-9.6$ days; $P=0.008$ ), higher rate of colectomy within 3 months of admission (OR, 5.5; 95\% CI, 1.1-28.2; $P=0.042$ ) and more frequent ICU admissions (OR, 7.8; 95\% CI, 1.5-41.6; $P=0.017$ ). Thirty- and 90-day readmission rates were lower in the cohort that received CS postCDI diagnosis (OR, 0.2; 95\% CI, 0.0-1.0; $P=0.047$ and OR, 0.3; 95\% CI, 0.1-1.0; $P=0.040$, respectively) (Table 6). Pre-CDI diagnosis CS use was associated with an increase in 90-day readmission (OR, 3.3 95\% CI, 1.2-9.2; $P=0.025$ ) but was not associated with length of hospital stay, 30-day readmission, col- ectomy within 3 months of admission, ICU admission or CDI relapse. Inpatient immunomodulator/biologic use was not associated with any of the adverse outcomes. The number of endpoints for colon perforation and death were too small for propensity analysis (Table 6).

\section{5) Propensity-Adjusted Regression Analysis of Post-CDI Diagnosis CS Use Based on Types of IBD}

CS use post-CDI diagnosis in UC patients was associated with prolonged hospitalization (6.2 days: $95 \%$ CI, $0.4-12.0$ days; $P=$ 0.036 ) and more frequent ICU admissions (OR, 7.4; 95\% CI, 1.1-48.7; $P=0.036$ ), but was not associated with 30 - and 90day readmission, colectomy within 3 months of admission and CDI relapse. Post-CDI diagnosis CS use in CD patients was not associated with any of the adverse outcomes. The number of endpoints for 30-day readmission and ICU admission were too small for propensity analysis in the CD group (Table 7).

Table 6. Outcomes: Propensity-Adjusted Regression Analysis

\begin{tabular}{|c|c|c|c|c|c|c|}
\hline \multirow[t]{2}{*}{ Outcome } & \multicolumn{2}{|c|}{$\begin{array}{c}\text { Received CS pre-CDI } \\
\text { diagnosis }\end{array}$} & \multicolumn{2}{|c|}{$\begin{array}{c}\text { Received CS post-CDI } \\
\text { diagnosis }\end{array}$} & \multicolumn{2}{|c|}{$\begin{array}{l}\text { Inpatient Immunomodulator/ } \\
\text { biologic use }\end{array}$} \\
\hline & OR $(95 \% \mathrm{Cl})$ & $P$-value & OR $(95 \% \mathrm{Cl})$ & $P$-value & OR $(95 \% \mathrm{Cl})$ & $P$-value \\
\hline Length of stay (day) & $1.0(-3.07$ to 5.10$)$ & 0.624 & 5.5 (1.45 to 9.56$)$ & $0.008^{\mathrm{a}}$ & $0.3(-2.92$ to 3.61$)$ & 0.835 \\
\hline 30-Day readmission & 3.2 (0.89 to 11.77$)$ & 0.074 & 0.2 (0.03 to 0.98$)$ & $0.047^{\mathrm{a}}$ & 2.3 (0.70 to 7.52$)$ & 0.169 \\
\hline 90-Days readmission & 3.3 (1.16 to 9.17$)$ & $0.025^{a}$ & 0.3 (0.09 to 0.94$)$ & $0.040^{\mathrm{a}}$ & 1.4 (0.56 to 3.39 ) & 0.478 \\
\hline Colectomy within 3 months of admission & $1.9(0.32$ to 10.84$)$ & 0.483 & 5.5 (1.07 to 28.24) & $0.042^{\mathrm{a}}$ & 0.6 (0.15 to 2.08$)$ & 0.381 \\
\hline ICU admission & $0.9(0.15$ to 5.15$)$ & 0.894 & 7.8 (1.45 to 41.57$)$ & $0.017^{\mathrm{a}}$ & 0.2 (0.04 to 0.96$)$ & 0.054 \\
\hline CDI relapse & 1.4 (0.46 to 4.04$)$ & 0.568 & 0.5 (0.16 to 1.74$)$ & 0.297 & 0.8 (0.30 to 1.95$)$ & 0.577 \\
\hline
\end{tabular}

${ }^{a}$ Significant $P$-value.

CS, corticosteroid; CDI, Clostridium difficile infection; ICU, intensive care unit.

Table 7. Outcomes: Propensity-Adjusted Regression Analysis of CS Use Post-CDI Diagnosis Based on Types of IBD

\begin{tabular}{|c|c|c|c|c|}
\hline \multirow{3}{*}{ Outcome } & \multicolumn{4}{|c|}{ Received CS post-CDI diagnosis } \\
\hline & \multicolumn{2}{|l|}{ UC } & \multicolumn{2}{|c|}{$\mathrm{CD}$} \\
\hline & OR $(95 \% \mathrm{Cl})$ & $P$-value & OR $(95 \% \mathrm{Cl})$ & $P$-value \\
\hline Length of stay (day) & 6.2 (0.41 to 12.01$)$ & $0.036^{\mathrm{a}}$ & $1.6(-3.88$ to 7.16$)$ & 0.553 \\
\hline 30-Day readmission & 0.4 (0.05 to 2.92$)$ & 0.356 & NA & \\
\hline 90-Days readmission & 0.4 (0.09 to 1.91$)$ & 0.264 & 0.2 (0.02 to 1.18$)$ & 0.072 \\
\hline Colectomy within 3 months of admission & 10.8 (0.79 to 148.42$)$ & 0.074 & 3.54 (0.12 to 80.52$)$ & 0.427 \\
\hline ICU admission & 7.4 (1.14 to 48.67) & $0.036^{\mathrm{a}}$ & NA & \\
\hline CDI relapse & 0.2 (0.01 to 3.28$)$ & 0.273 & 0.9 (0.21 to 3.68$)$ & 0.857 \\
\hline
\end{tabular}

${ }^{a}$ Significant $P$-value.

CS, corticosteroid; CDI, Clostridium difficile infection; ICU, intensive care unit; NA, not available. 


\section{6) Propensity-Adjusted Regression Analysis of Post-CDI Diagnosis CS Use Based on CS Doses}

We performed a sensitivity analyses accounting for dose ranges, and demonstrated no differences in the association of receiving CS following CDI diagnosis on length of stay, 30-day readmission, 90-day readmission and CDI relapse. Colectomy and ICU stay outcomes were too small for analysis.

\section{DISCUSSION}

In the present study, we have demonstrated that patients who received CS for presumed IBD exacerbation following a diagnosis of CDI by PCR had an increased length of hospital stay, higher rate of ICU admission and increased rate of colectomy by propensity-adjusted regression analysis. Pre-CDI diagnosis CS use alone was not associated with negative outcomes. The wide confidence intervals with patients at higher risk of colectomy and ICU admission are in keeping with the small number of outcomes in this cohort. When stratified by IBD type, CS post-CDI diagnosis was not associated with adverse outcomes in the CD group but was associated with an increased length of stay and ICU admission in UC patients. Propensityadjusted regression analysis was not performed for immunomodulator/biologic use for subgroups of UC and CD as the outcomes were too small for analysis.

Given the paucity of available data, there is significant variability in treatment practices for patients with IBD flares and CDI. ${ }^{24}$ In a survey of 169 North American Gastroenterologists, $46 \%$ elected to add immunosuppressive medications when dealing with CDI versus $54 \%$ who elected to treat the infection with antibiotics alone. ${ }^{24}$ Professional society guidelines have had difficulty filling this gap and standardizing treatment. The European Crohn's and Colitis Organisation guidelines for management of opportunistic infections in IBD from 2014, similar to the more general CD and UC guidelines from 2016, recommend CDI testing of all patients with IBD flare. The authors report that immunomodulators and especially CS may be associated with CDI development and negative outcomes in IBD, but were unable to offer a specific strategy for their management. ${ }^{25,26}$ The recent American Gastroenterological Association clinical practice update suggests that clinicians may postpone escalation of CS and other immune therapies in the setting of acute CDI, but states that there is little evidence available to provide a firm recommendation. ${ }^{10}$

Ben-Horin et al. ${ }^{16}$ evaluated the effects of combination therapy with antibiotics and immunomodulators on patients hos- pitalized with IBD and CDI diagnosed by toxin assay. A total of 155 patients (59\% with UC) all of whom were treated with antibiotics for CDI, with $67 \%$ also receiving immunomodulators, defined by use of CS at a dose of $20 \mathrm{mg}$ of prednisone or greater, thiopurines, methotrexate, cyclosporine, tacrolimus or biologics of any kind. The authors observed a trend for negative outcomes such as death and colectomy when immunomodulators were used (likelihood ratio, 11.9; 95\% Cl, 0.9-157; $P=0.06$ ), but that only combinations of 2 or 3 immunomodulators were correlated with these negative outcomes on multivariate analysis (OR, $17 ; 95 \% \mathrm{Cl}, 3.2-91 ; P<0.01) .{ }^{16}$ More recently, Ananthakrishnan et al., ${ }^{27}$ utilizing clinical markers of disease severity similar to our own, examined a group of 294 hospitalized patients with both IBD and CDI identified by ICD9 code search. While 58 patients (19.7\%) met the primary outcome of colectomy $(n=45)$ or death $(n=13)$, only albumin levels below $3 \mathrm{~g} / \mathrm{dL}$ were significantly associated with these outcomes, but not medication use including CS, azathioprine, 6-mercaptopurine, methotrexate, infliximab, adalimumab within 30 days after or before the diagnosis of CDI. Given the study period January 1998 through June 2010, it seems likely that very few of these patients had their diagnosis made by PCR testing.

While we have shown an association between CS use and adverse outcomes, limitations of the retrospective study design prevent drawing any stronger conclusions of a direct cause and effect relationship. Our main limiting factor, common to other retrospective work analyzing IBD populations, was the lack of standard IBD clinical activity scores to allow for additional analysis of outcomes by disease severity. Also, with only $21 \%$ of our own patients having either colonoscopy or sigmoidoscopy, we were unable to analyze outcomes by endoscopic disease severity or phenotype. Though surrogate markers of disease severity used in other recent retrospective studies of IBD and C. difficile ${ }^{16,27}$ were similar for those receiving CS and those not, it would still be reasonable to assume a bias towards prescribing CS in those with more severe clinical presentations who would be expected to be at higher risk of negative outcomes.

Gaps in the medical record, as well the small number of endoscopic procedures also prevented analysis by IBD clinical characteristics beyond the diagnosis of CD or UC. Additional bias would include the lack of a protocol directing the choice of antibiotic therapy prescribed, though the analysis found no gross difference in antibiotics used between those receiving and not receiving CS. 
It is important to note that the decision to begin CS was made for almost all patients prior to or immediately following their CDI diagnosis, that is, patients receiving CS following CDI diagnosis were generally not given an opportunity to recover with a trial of antibiotic therapy alone, as recent expert opinion has recommended. ${ }^{28}$ Also, we observed a significant decline in 30- and 90-day readmission rates in those who did receive CS and avoided colectomy, suggesting that some subgroup of patients may benefit from CS use. It is possible that this may be due to a group of patients for whom the main acute issue was the IBD rather than the CDI, that is, those truly colonized rather than infected. These mixed results would seem to add support to current guidelines advocating a 2-step process, incorporating toxin assay, for the diagnosis of CDI in the setting of IBD, ${ }^{10}$ where loose stools even without true infection are so common. Also, while readmission to the 2 health systems among those diagnosed with CDI is very closely tracked, we cannot rule out the possibility that patients may have been admitted to facilities outside of the Northwell Health and Stony Brook University Hospital Systems following their initial discharge.

In conclusion, we have demonstrated that among hospitalized patients with UC and PCR diagnosed CDI, post-CDI diagnosis CS use was associated with an increased risk of colectomy, ICU admission and prolonged hospitalization. Our findings suggest that CS use among hospitalized UC patients with PCR diagnosed C. difficile is associated with negative outcomes, and would seem to reinforce the importance of $C$. difficile toxin assay to help distinguish colonization from infection. The findings also further support the utilization of a trial of antibiotic therapy prior to CS use. While these findings do not preclude the use of other immune therapies, including the continuation of outpatient medications following hospitalization, the results do suggest that CS should be used cautiously in this population.

\section{FINANCIAL SUPPORT}

The authors received no financial support for the research, authorship, and/or publication of this article.

\section{CONFLICT OF INTEREST}

No potential conflict of interest relevant to this article was reported.

\section{AUTHOR CONTRIBUTION}

Lim HW and Sultan K designed the study, collected, analyzed and interpreted the data. Lim HW, Schuster IP, and Sultan K performed the literature review and wrote the article. Rajapakse R, Monzur F, and Sultan K critically revised the manuscript. Khan S provided administrative and material support. Lim HW is the article guarantor. Approval of final manuscript: all authors.

\section{ACKNOWLEDGEMENTS}

We thank Kay Chen, BS of Stony Brook University Hospital for assistance with data collection.

\section{REFERENCES}

1. Tamboli CP, Neut C, Desreumaux P, Colombel JF. Dysbiosis in inflammatory bowel disease. Gut 2004;53:1-4.

2. Dupaul-Chicoine J, Dagenais M, Saleh M. Crosstalk between the intestinal microbiota and the innate immune system in intestinal homeostasis and inflammatory bowel disease. Inflamm Bowel Dis 2013;19:2227-2237.

3. MacDonald TT, Monteleone G. Overview of role of the immune system in the pathogenesis of inflammatory bowel disease. Adv Exp Med Biol 2006;579:98-107.

4. Rubin MS, Bodenstein LE, Kent KC. Severe Clostridium difficile colitis. Dis Colon Rectum 1995;38:350-354.

5. Lamontagne F, Labbé AC, Haeck O, et al. Impact of emergency colectomy on survival of patients with fulminant Clostridium difficile colitis during an epidemic caused by a hypervirulent strain. Ann Surg 2007;245:267-272.

6. Kelly CP, LaMont JT. Clostridium difficile: more difficult than ever. N Engl J Med 2008;359:1932-1940.

7. Gabriel L, Beriot-Mathiot A. Hospitalization stay and costs attributable to Clostridium difficile infection: a critical review. J Hosp Infect 2014;88:12-21.

8. Walker AS, Eyre DW, Wyllie DH, et al. Characterisation of Clostridium difficile hospital ward-based transmission using extensive epidemiological data and molecular typing. PLoS Med 2012;9:e1001172.

9. Lessa FC, Mu Y, Bamberg WM, et al. Burden of Clostridium difficile infection in the United States. N Engl J Med 2015;372: 825-834.

10. Khanna S, Shin A, Kelly CP. Management of Clostridium difficile infection in inflammatory bowel disease: expert review 
from the Clinical Practice Updates Committee of the AGA Institute. Clin Gastroenterol Hepatol 2017;15:166-174.

11. Bossuyt P, Verhaegen J, Van Assche G, Rutgeerts P, Vermeire S. Increasing incidence of Clostridium difficile-associated diarrhea in inflammatory bowel disease. J Crohns Colitis 2009;3: 4-7.

12. Rodemann JF, Dubberke ER, Reske KA, Seo DH, Stone CD. Incidence of Clostridium difficile infection in inflammatory bowel disease. Clin Gastroenterol Hepatol 2007;5:339-344.

13. Khanna S, Pardi DS. IBD: poor outcomes after Clostridium difficile infection in IBD. Nat Rev Gastroenterol Hepatol 2012; 9:307-308.

14. Ananthakrishnan AN, McGinley EL, Binion DG. Excess hospitalisation burden associated with Clostridium difficile in patients with inflammatory bowel disease. Gut 2008;57:205210.

15. Ananthakrishnan AN, McGinley EL, Saeian K, Binion DG. Temporal trends in disease outcomes related to Clostridium difficile infection in patients with inflammatory bowel disease. Inflamm Bowel Dis 2011;17:976-983.

16. Ben-Horin S, Margalit M, Bossuyt P, et al. Combination immunomodulator and antibiotic treatment in patients with inflammatory bowel disease and Clostridium difficile infection. Clin Gastroenterol Hepatol 2009;7:981-987.

17. Issa M, Vijayapal A, Graham MB, et al. Impact of Clostridium difficile on inflammatory bowel disease. Clin Gastroenterol Hepatol 2007;5:345-351.

18. Razik R, Rumman A, Bahreini Z, McGeer A, Nguyen GC. Recurrence of Clostridium difficile infection in patients with inflammatory bowel disease: the RECIDIVISM Study. Am J Gastroenterol 2016;111:1141-1146.

19. Sunkesula VC, Kundrapu S, Muganda C, Sethi AK, Donskey CJ. Does empirical Clostridium difficile infection (CDI) therapy result in false-negative CDI diagnostic test results? Clin Infect Dis 2013;57:494-500.
20. Leffler DA, Lamont JT. Clostridium difficile Infection. N Engl J Med 2015;373:287-288.

21. Bagdasarian N, Rao K, Malani PN. Diagnosis and treatment of Clostridium difficile in adults: a systematic review. JAMA 2015; 313:398-408.

22. Crobach MJ, Planche T, Eckert C, et al. European Society of Clinical Microbiology and Infectious Diseases: update of the diagnostic guidance document for Clostridium difficile infection. Clin Microbiol Infect 2016;22 Suppl 4:S63-S81.

23. Planche TD, Davies KA, Coen PG, et al. Differences in outcome according to Clostridium difficile testing method: a prospective multicentre diagnostic validation study of $\mathrm{C}$ difficile infection. Lancet Infect Dis 2013;13:936-945.

24. Yanai H, Nguyen GC, Yun L, et al. Practice of gastroenterologists in treating flaring inflammatory bowel disease patients with Clostridium difficile: antibiotics alone or combined antibiotics/immunomodulators? Inflamm Bowel Dis 2011;17:15401546.

25. Rahier JF, Magro F, Abreu C, et al. Second European evidencebased consensus on the prevention, diagnosis and management of opportunistic infections in inflammatory bowel disease. J Crohns Colitis 2014;8:443-468.

26. Magro F, Gionchetti P, Eliakim R, et al. Third European evidencebased consensus on diagnosis and management of ulcerative colitis. Part 1: definitions, diagnosis, extra-intestinal manifestations, pregnancy, cancer surveillance, surgery, and ileo-anal pouch disorders. J Crohns Colitis 2017;11:649-670.

27. Ananthakrishnan AN, Guzman-Perez R, Gainer V, et al. Predictors of severe outcomes associated with Clostridium difficile infection in patients with inflammatory bowel disease. Aliment Pharmacol Ther 2012;35:789-795.

28. Rao K, Higgins PD. Epidemiology, diagnosis, and management of Clostridium difficile infection in patients with inflammatory bowel disease. Inflamm Bowel Dis 2016;22:1744-1754. 